\title{
Trapped Phonons Improve Photon Detection
}

\section{Researchers more than double the frequency resolution of a superconducting single-photon detector through phonon trapping.}

\section{By Marric Stephens}

I hen a photon strikes a superconductor, its energy cascades through the material via electron-phonon interactions, generating thousands of excitations called quasiparticles. Before this energy dissipates, these quasiparticles measurably change the kinetic inductance of the material, a property that researchers have used to make superconducting single-photon detectors (see Synopsis: Direct Detection of Quasiparticles). Now, Pieter de Visser at the Netherlands Institute for Space Research and colleagues have modified the design of such a detector to achieve a 2.5-fold increase in the precision with which the device can measure photon frequency [1]. That precision increase could improve spectroscopy measurements of exoplanet atmospheres and fluorescence measurements of biological samples.

Currently, operational superconductor-based photon detectors are made as follows: A series of superconducting circuits is deposited on a thick ( $>300 \mu \mathrm{m}$ ) silicon or sapphire substrate, with each circuit making up one pixel. The frequency resolution

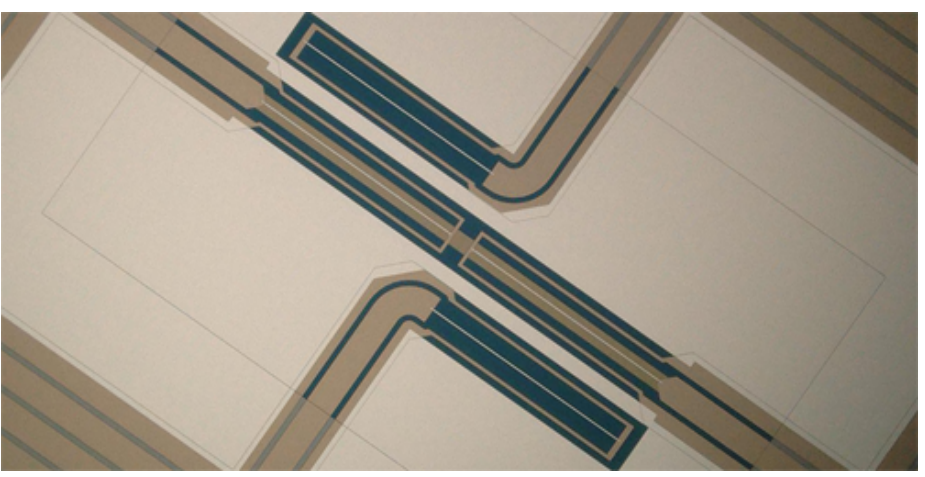

Credit: P. J. de Visser et al. [1] of these pixels is limited, because phonons can escape from the superconducting wire into the substrate, taking with them some of the incident photon's energy. This energy loss increases the statistical variance of the kinetic-inductance signal used to detect a photon, broadening the measured spectrum.

In their redesigned device, de Visser and his colleagues replace the substrate with a thin $(110 \mathrm{~nm})$ silicon-nitride membrane. They show that phonons escaping from the superconducting wire into this membrane reflect from the membrane's bottom surface back into the superconductor, where they excite more quasiparticles. Testing their setup, the researchers achieved resolving powers of 52 and 19 for optical and near-infrared photons, respectively. For conventional devices these numbers were 21 and 10. They now plan to scale up their device to create instruments suitable for astronomical and biological applications.

Marric Stephens is a Corresponding Editor for Physics based in Bristol, UK.

\section{REFERENCES}

1. P. J. de Visser et al., "Phonon-trapping-enhanced energy resolution in superconducting single-photon detectors," Phys. Rev. Applied 16, 034051 (2021). 